\title{
Advance notice of contraceptive availability at surgical abortion: a pilot randomised controlled trial
}

\author{
Andrea H Roe, ${ }^{1,2,3}$ Jennifer Fortin, ${ }^{3}$ Danielle Gelfand, ${ }^{3}$ \\ Elizabeth Janiak, ${ }^{1,2,3}$ Rie Maurer, $^{4}$ Alisa Goldberg ${ }^{1,2,3}$
}

\begin{abstract}
'Department of Obstetrics and Gynecology, Penn Medicine, Philadelphia, Pennsylvania, USA ${ }^{2}$ Harvard Medical School, Boston, Massachusetts, USA

${ }^{3}$ Planned Parenthood League of Massachusetts, Boston,

Massachusetts, USA

${ }^{4}$ Center for Clinical Investigation, Brigham and Women's Hospital, Boston, Massachusetts, USA
\end{abstract}

\section{Correspondence to}

Dr Andrea H Roe, Department of Obstetrics and Gynecology, Penn Medicine, Philadelphia, PA 19104,USA; andrea.roe@uphs. upenn.edu

Received 4 November 2017 Revised 24 April 2018 Accepted 1 May 2018 Published Online First 18 May 2018

\begin{abstract}
Background With advance notice about the availability and effectiveness of contraceptive methods, abortion patients have more time and information for decision-making. We assessed the impact of an informational telephone call prior to the surgical abortion visit on patient contraceptive knowledge.
\end{abstract}

Methods This was a pilot randomised controlled trial. Prior to their abortion visit, participants were randomised to the intervention message, a standardised notification about the availability, effectiveness and safety of long-acting (LARC) and short-acting reversible contraception (SARC) on the day of the abortion, or to the control message, a reiteration of appointment logistics without information about contraception. At the visit, participants completed a pre-procedure survey to assess contraceptive knowledge and usefulness of the intervention. The primary outcome was knowledge of LARC availability immediately after surgical abortion. A secondary outcome was contraceptive method uptake. Results We enrolled 234 subjects. The pre-visit telephone notification improved knowledge that LARC is available immediately after surgical abortion (71.3\% vs $50.9 \%, \mathrm{P}<0.01)$. Participants in both study arms found the telephone notifications useful. Post-abortion contraceptive method choice did not differ between study arms.

Conclusions Advance notice about contraception was acceptable to surgical abortion patients and improved their contraceptive knowledge.

Trial registration number NCT02836561.

\section{INTRODUCTION}

Abortion represents an important opportunity for women to choose contraception. Long-acting reversible contraception (LARC), including the intrauterine device (IUD) and the subdermal implant, and short-acting reversible contraception

\section{Key messages}

- Surgical abortion patients underestimate the availability and safety of immediate post-abortion contraception and overestimate the effectiveness of shortacting hormonal contraception.

- A pre-visit informational telephone call improved surgical patients' knowledge that long-acting reversible contraception was available at the time of their procedure.

- Advance notice of contraceptive availability and effectiveness can be integrated into routine abortion care to optimise contraceptive decision-making.

(SARC), including the oral contraceptive pill, transdermal patch, vaginal ring and progestin injection, may be started immediately after surgical abortion. ${ }^{1}$ Since both ovulation and sexual activity usually resume within several weeks, ${ }^{2}{ }^{3}$ pairing contraception with abortion ensures continuous protection against pregnancy. The majority of women seeking abortion want and expect to obtain contraception at their visit. ${ }^{4}$

The IUD and implant have the highest contraceptive effectiveness of all reversible methods. Women who receive a LARC device at the time of abortion are significantly less likely to have another abortion within 2 years compared with women who choose other methods. ${ }^{5} 6$ Women who desire an IUD are more likely to obtain one via immediate post-abortion insertion compared with interval placement, since only one-third of those who intend to follow-up for a post-abortion IUD do. ${ }^{478}$ For women who receive immediate post-abortion LARC, continuation rates are high. ${ }^{9-11}$ 
Despite the effectiveness of LARC and convenience of post-abortion placement, only $6.6 \%$ of surgical abortion patients in the USA received immediate post-procedure LARC, according to a 2009 survey by the National Abortion Federation. ${ }^{12}$ Structural barriers, such as clinic flow, device stocking, and insurance reimbursement, have historically limited access to post-abortion LARC. ${ }^{12} 13$ In addition, patients may be poorly informed about the availability, safety and effectiveness of LARC. ${ }^{14} 15$

In the USA, contraceptive counselling often occurs on the same day as the abortion procedure (when state law does not require a waiting period). Counselling during the abortion appointment holds potential to increase contraceptive knowledge and uptake, but strategies have had mixed results, ${ }^{16}$ and some patients do not want to discuss contraception at the time of abortion. ${ }^{17}$ For women with limited previous knowledge about contraception, counselling on the day of abortion may not provide enough time to fully consider various methods, and some women may feel too emotionally burdened to adequately address contraception. Therefore, providing information about contraception before the clinical visit might allow patients more time and freedom to reach a better-informed choice.

This study piloted a brief pre-visit contraceptive information intervention delivered via telephone. We hypothesised that advance notice of post-abortion contraceptive options would result in greater knowledge about contraception, particularly LARC, with an aim toward improving patients' ability to choose and initiate the optimal method for themselves.

\section{METHODS}

We conducted a pilot randomised controlled trial (RCT) of a pre-visit intervention for women who scheduled a surgical abortion appointment between August 2016 and April 2017 at the Boston site of Planned Parenthood League of Massachusetts (PPLM-Boston). This study was approved by the Partners Human Research Committee. At PPLM, patients call and schedule their own abortion procedure. No referrals or pre-operative visits are required, and patients receive abortion and contraceptive counselling and their abortion procedure and contraceptive method (if desired) on the same day. All surgical abortion patients are screened for insurance coverage of LARC in advance of their appointment, and most can obtain same-day LARC coverage through their public or private insurance. Counsellors routinely offer LARC along with other methods during the pre-procedure information session, and providers verify contraceptive choice at the time of the abortion. All physicians are trained in immediate post-procedure LARC insertion. Surgical abortions take place in an outpatient clinic setting with intravenous sedation or a paracervical block alone for anaesthesia. All SARC methods are also available and offered on the same day.
We designed this as a pilot to test the feasibility and utility of delivering a telephone message about contraception to abortion patients in advance of their appointment. Our primary outcome was patient knowledge that LARC is available for insertion at the same time as surgical abortion. We chose this outcome in order to assess whether participants retained the information that we provided in our intervention. Secondary outcomes included knowledge of post-abortion LARC safety, knowledge of LARC effectiveness, perceived usefulness of the telephone notification, method of post-abortion contraception, and perceived contraceptive autonomy.

In the absence of information about patients' baseline knowledge about post-abortion contraception, we used our clinical experience to estimate that $30 \%$ of women in the control arm would know that LARC devices were available for immediate post-abortion insertion. We conservatively projected an increase to $50 \%$ as a result of the intervention, knowing that, although we would be telling $100 \%$ of women in the intervention arm that they could safely receive post-abortion LARC, some might not remember or believe the message. Assuming $80 \%$ power and two-sided alpha 0.05 , we required 186 subjects. To account for $20 \%$ attrition and missing data, we planned to enroll 234 subjects in total.

Patients scheduled for a surgical abortion procedure who agreed to receive a telephone call from research staff were offered participation in the study. Subjects needed to be 18 years of age or older and to speak English proficiently. After describing the risks and benefits of the study, research staff consented subjects verbally over the telephone, then enrolled and randomised them to an intervention or control message. We used a computer-generated 1:1 random allocation sequence with block sizes of 13 and concealed the assignments in sequentially numbered opaque envelopes. The research assistant opened these envelopes after enrolling each subject and delivered an intervention or control script, each less than 1 minute long, depending on the randomisation assignment.

The intervention script notified participants that all reversible methods were available and safe to start on the day of surgical abortion (see online supplementary appendix). The research assistant presented LARC methods first, describing them as the most effective forms of contraception, and SARC methods second, without explicit mention of their effectiveness. We chose to emphasise LARC methods due to their superior effectiveness and because patients know less about the effectiveness of LARC than SARC. ${ }^{18}$ No individualised counselling was performed during this telephone call, and participants were told they could discuss contraception further at their appointment. Specific questions were directed to the clinic's counselling hotline. The control script was a brief reminder about the logistics of the appointment, such as anticipated time in clinic, with no information about 
contraception. Participants and clinical staff were blinded to the subjects' intervention status.

On arrival at the clinic, and prior to the contraceptive counselling session and procedure, subjects completed a self-administered electronic survey that assessed the primary outcome, knowledge that LARC is available to start at time of surgical abortion. The survey also assessed usefulness of the pre-visit telephone call, general knowledge of contraceptive effectiveness and safety, and demographic, social and reproductive history.

After the abortion, subjects completed a second survey, which included a modified Health Care Climate Questionnaire (mHCCQ), a six-item questionnaire in which patients rated how well their healthcare team supported their autonomy on a scale from 1 (low) to 7 (high), which has been validated for use in abortion care (Sznajder et al, 2018 unpublished data). The purpose of this assessment was to evaluate whether advance notice about contraception positively or negatively impacted participants' perception of their contraceptive autonomy. We wanted to ensure that this pilot intervention would not be construed as undue pressure to use contraception or a specific method.

After the visit, research staff collected data from the subject's electronic medical record, including ultrasound-confirmed gestational age and post-abortion contraceptive method received. We defined 'contraceptive method received' as a device or injection inserted immediately post-abortion or a prescription given for a pill, patch or ring.

Intent-to-treat analysis was performed. Numerical demographic and clinical variables were compared between study arms using Student's t-test and the Wilcoxon rank sum test. Categorical demographic and clinical variables were compared using chi-square and Fisher's exact tests. Chi-square and Fisher's exact tests were also used to compare categorical data between intervention and control groups for the primary and secondary outcomes. The mHCCQ score was calculated as a mean of the six-item survey and compared between arms using the Wilcoxon rank sum test. Adjusted analysis of the primary outcome was performed using logistic regression. All statistical analysis was done using SAS v. 9.4 (Cary, NC).

\section{Patient involvement}

We designed the study intervention to maximise patients' opportunity to choose same-day contraception. Previous studies have shown that most patients want contraception on the same day as their abortion, but clinics do not always meet patient need. ${ }^{41213}$ We hypothesised that, in addition to well-established structural barriers, a lack of familiarity or knowledge of contraceptive methods, especially LARC, might impede patient access. Our primary and most secondary outcomes therefore measured the impact of this intervention on patients' contraceptive knowledge.

Before implementing the study, we tested our survey on five pilot subjects to ensure question clarity and ease of completion. The pilot did not raise any issues and therefore we did not alter the study protocol. In order to measure the burden and any inadvertent coercive effect of the pre-visit contraception message, we asked participants to evaluate the utility of the telephone call and their contraceptive autonomy.

\section{RESULTS}

Of 979 women with surgical abortion appointments during the study period, 271 women agreed to be contacted by the research team. We called these 271 women to offer study participation and enrolled our goal of 234 subjects (figure 1). Attrition was less than estimated (6.8\%), and 218 subjects completed the pre-abortion survey. Participants did not differ significantly between study arms in age, race, ethnicity, relationship status, gestational age, previous birth or

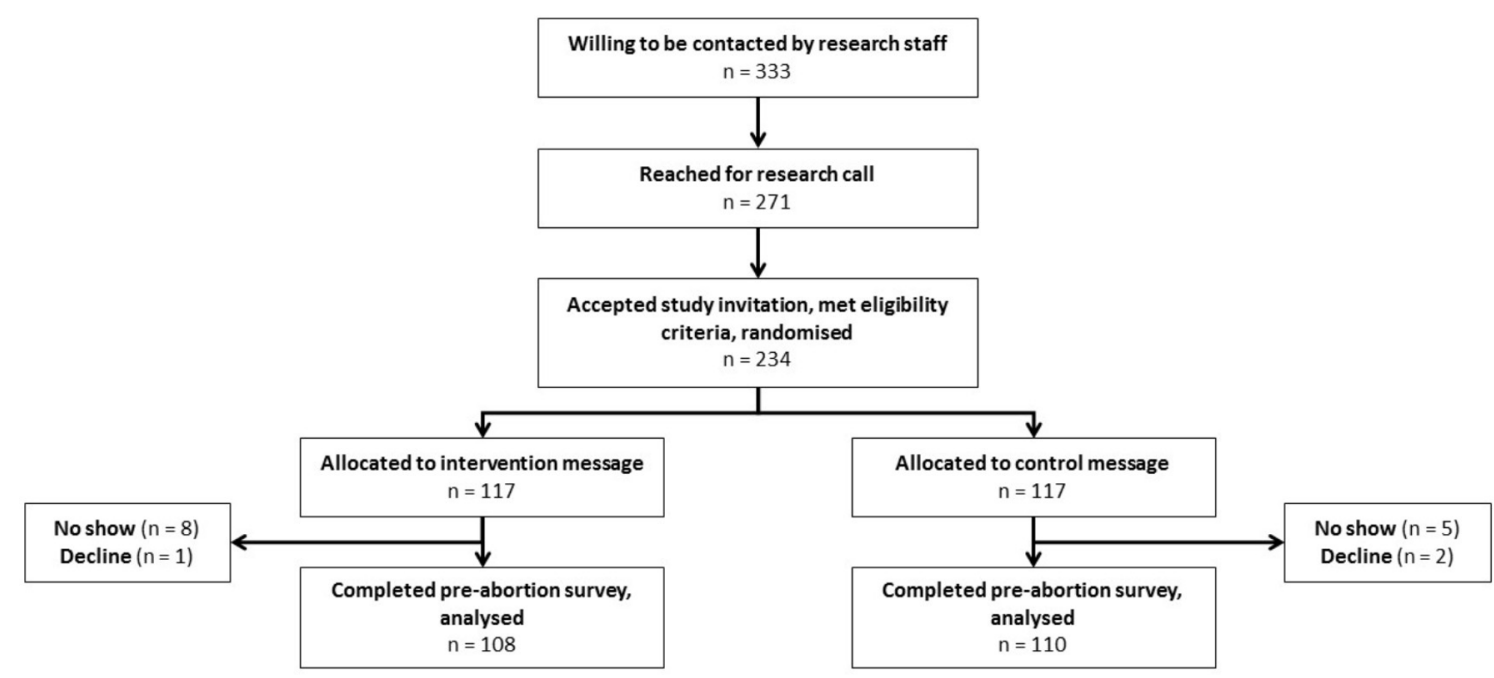

Figure 1 Participant flow diagram, per Consolidated Standards of Reporting Trials. 
Table 1 Baseline demographic characteristics of study participants

\begin{tabular}{|c|c|c|c|}
\hline Characteristic & $\begin{array}{l}\text { Intervention } \\
\operatorname{arm}(n=117) \\
{[n(\%)]}\end{array}$ & $\begin{array}{l}\text { Control } \\
\operatorname{arm}(n=117) \\
{[n(\%)]}\end{array}$ & $P$ values \\
\hline Age (years) & $\begin{array}{l}26.1 \pm 5.3 \\
(n=107)\end{array}$ & $\begin{array}{l}25.9 \pm 4.9 \\
(n=109)\end{array}$ & 0.77 \\
\hline Race & & & 0.10 \\
\hline Black & $27(23.1)$ & $32(27.4)$ & \\
\hline White & $34(29.0)$ & $43(36.8)$ & \\
\hline Asian & $4(3.4)$ & $9(7.7)$ & \\
\hline Mix & $7(6.0)$ & $8(6.8)$ & \\
\hline Other & $12(10.3)$ & $6(5.1)$ & \\
\hline No information & $33(28.2)$ & $19(16.2)$ & \\
\hline Hispanic or Latina & & & 0.55 \\
\hline No & $90(76.9)$ & $88(75.2)$ & \\
\hline Yes & $17(14.5)$ & $22(18.8)$ & \\
\hline No information & $10(8.6)$ & $7(6.0)$ & \\
\hline Education & & & 0.01 \\
\hline $\begin{array}{l}\text { Some high } \\
\text { school }\end{array}$ & $2(1.7)$ & $14(12.0)$ & \\
\hline $\begin{array}{l}\text { High school or } \\
\text { equivalent }\end{array}$ & $33(28.2)$ & $21(17.9)$ & \\
\hline $\begin{array}{l}\text { Some college } \\
\text { or associates } \\
\text { degree }\end{array}$ & $46(39.3)$ & $48(41.0)$ & \\
\hline $\begin{array}{l}\text { College graduate } \\
\text { or more }\end{array}$ & 27 (23.1) & $27(23.1)$ & \\
\hline No information & $9(7.7)$ & $7(6.0)$ & \\
\hline Insurance & $9(7.7)$ & & 0.06 \\
\hline Public & $60(51.3)$ & $69(59.0)$ & \\
\hline Private & $29(24.8)$ & $22(18.8)$ & \\
\hline Out of pocket & $24(20.5)$ & $24(20.5)$ & \\
\hline $\begin{array}{l}\text { Financial } \\
\text { assistance }\end{array}$ & $2(1.7)$ & $0(0.0)$ & \\
\hline Other & $2(1.7)$ & $2(1.7)$ & \\
\hline $\begin{array}{l}\text { Gestational age } \\
\text { (days) }\end{array}$ & $\begin{array}{l}58[47,76] \\
n=105\end{array}$ & $\begin{array}{l}57[47,72] \\
n=106\end{array}$ & 0.70 \\
\hline Parity & & & 0.63 \\
\hline 0 & $59(50.4)$ & $52(44.4)$ & \\
\hline$\geq 1$ & $48(41.0)$ & $55(47.0)$ & \\
\hline No information & $10(8.6)$ & $10(8.6)$ & \\
\hline $\begin{array}{l}\text { Previous } \\
\text { abortion }\end{array}$ & & & 0.13 \\
\hline 0 & $38(32.5)$ & $53(45.3)$ & \\
\hline$\geq 1$ & $69(59.0)$ & $56(47.9)$ & \\
\hline No information & $10(8.5)$ & $8(6.8)$ & \\
\hline $\begin{array}{l}\text { Regular male } \\
\text { partner }\end{array}$ & & & 0.50 \\
\hline No & $21(18.0)$ & $28(23.9)$ & \\
\hline Yes & $87(74.3)$ & $82(70.1)$ & \\
\hline No information & $9(7.7)$ & $7(6.0)$ & \\
\hline
\end{tabular}

Table 2 Contraceptive knowledge and uptake, intervention usefulness, and perception of contraceptive autonomy among study participants.

\begin{tabular}{|c|c|c|c|}
\hline & $\begin{array}{l}\text { Intervention } \\
\text { message } \\
(n=108)[n(\%)]\end{array}$ & $\begin{array}{l}\text { Control message } \\
(n=110)[n(\%)]\end{array}$ & $P$ values \\
\hline \multicolumn{4}{|c|}{$\begin{array}{l}\text { Which birth control methods are available for you to start after your } \\
\text { abortion today? }\end{array}$} \\
\hline $\begin{array}{l}\text { LARC } \\
\text { SARC }\end{array}$ & $\begin{array}{l}77(71.3) \\
90(83.3)\end{array}$ & $\begin{array}{l}56(50.9) \\
88(80.0)\end{array}$ & $\begin{array}{l}<0.01 \\
0.53\end{array}$ \\
\hline \multicolumn{4}{|c|}{$\begin{array}{l}\text { Which birth control methods are over } 99 \% \text { effective at } \\
\text { preventing pregnancy? }\end{array}$} \\
\hline $\begin{array}{l}\text { LARC } \\
\text { SARC }\end{array}$ & $\begin{array}{l}74(68.5) \\
42(38.9)\end{array}$ & $\begin{array}{l}81(73.6) \\
89(80.9)\end{array}$ & $\begin{array}{l}0.40 \\
<0.01\end{array}$ \\
\hline \multicolumn{4}{|c|}{ Which birth control methods are safe for you to start today? } \\
\hline $\begin{array}{l}\text { LARC } \\
\text { SARC }\end{array}$ & $\begin{array}{l}61(56.5) \\
74(68.5)\end{array}$ & $\begin{array}{l}56(50.9) \\
84(76.4)\end{array}$ & $\begin{array}{l}0.41 \\
0.19\end{array}$ \\
\hline \multicolumn{4}{|c|}{ Contraception received or prescribed } \\
\hline $\begin{array}{l}\text { LARC } \\
\text { SARC }\end{array}$ & $\begin{array}{l}37(31.6) \\
36(30.8)\end{array}$ & $\begin{array}{l}40(34.2) \\
34(29.1)\end{array}$ & $\begin{array}{l}0.68 \\
0.78\end{array}$ \\
\hline \multicolumn{4}{|c|}{ Usefulness of message } \\
\hline $\begin{array}{l}\text { Not useful } \\
\text { Useful }\end{array}$ & $\begin{array}{l}2(1.9) \\
106(98.1)\end{array}$ & $\begin{array}{l}3(2.7) \\
107(97.3)\end{array}$ & 1.0 \\
\hline $\begin{array}{l}\text { Modified Health } \\
\text { Care Climate } \\
\text { score }\end{array}$ & $7.0(6.5,7.0)$ & $7.0(6.5,7.0)$ & 0.77 \\
\hline
\end{tabular}

Numeric variables are presented with median and IQR. Categorical variables are reported with frequency (\%).

LARC, long-acting reversible contraception; SARC, short-acting reversible contraception.

previous abortion (table 1). The groups differed in education, with more women in the intervention arm having completed high school, and more women in the control arm having completed only part of high school.

Numeric variables (age, gestational age) are presented with mean and SD or median and IQR. Categorical variables are reported with frequency (\%).

The intervention improved knowledge about the availability of LARC at the time of surgical abortion (table 2): $71.3 \%$ in the intervention arm knew that immediate post-abortion LARC was available, compared with $50.9 \%$ in the control arm $(\mathrm{P}<0.01)$. We used logistic regression to adjust for the baseline disparity in education level between study arms, and the primary outcome remained significantly different: unadjusted OR 2.40 [95\% CI 1.37-4.49], adjusted OR 2.62 (1.45-4.73). Knowledge about SARC availability was the same across study arms.

Knowledge about LARC effectiveness was the same in both study arms. However, despite the fact that the intervention message did not explicitly detail SARC effectiveness, subjects who received the intervention had significantly better knowledge about SARC effectiveness. Whereas $80.9 \%$ of women in the control arm incorrectly thought that SARC was over 99\% effective, only $38.9 \%$ of women in the intervention arm were 
under this misconception $(\mathrm{P}<0.01)$. The majority of women in both arms knew SARC was safe, while only half the women in both arms thought LARC was safe.

Post-abortion contraceptive choice was equivalent across study arms. One-third of women received a LARC method at the time of abortion and just under one-third of women received a SARC method or prescription on the day of the procedure. We do not have follow-up information for those women who planned to receive contraception from an outside provider.

Nearly all participants $(98.0 \%$ of the intervention arm and $97.2 \%$ of the control arm) felt that the telephone message was somewhat or very useful.

The median mHCCQ score in both study arms was 7 , indicating that both groups felt maximally supported by their healthcare providers and autonomous in their contraceptive decision-making.

\section{DISCUSSION}

Notifying women about contraceptive availability and effectiveness prior to their abortion appointment improved knowledge that LARC is available at time of abortion and that SARC is less than 99\% effective. In this pilot, advance notice of contraceptive availability and effectiveness successfully impacted patients' knowledge prior to the abortion appointment without compromising their autonomy.

Our intervention significantly improved knowledge of LARC availability. Although we underestimated baseline knowledge of LARC availability in our initial power calculation, we performed a post hoc power calculation utilising the observed LARC knowledge in the control arm (51\%) and confirmed that our sample size had $85 \%$ power to detect the $20 \%$ difference in LARC knowledge between arms. Women in the intervention arm also had significantly better knowledge of SARC effectiveness. Although we did not explicitly enumerate SARC effectiveness, women in the intervention arm may have inferred SARC's relatively lower effectiveness compared with LARC or they may have researched SARC after the telephone call. Alternatively, this difference in arms may reflect a baseline difference in knowledge.

This study also demonstrates that abortion patients have baseline misconceptions about SARC and LARC. Women in the control arm incorrectly believed that compared with LARC, SARC was more available and safer to initiate immediately post-abortion and was more effective at preventing pregnancy. These findings are consistent with previous research indicating that women often lack knowledge about the availability, effectiveness and safety of contraceptive methods, especially LARC. ${ }^{18}$

Since this study was designed as an RCT, participants had to give permission to be contacted by research staff and then received a research call that was separate from the clinical scheduling telephone encounter. Only
$28 \%$ of scheduled surgical abortion patients agreed to be contacted, therefore the study population may not be representative of the clinic population as a whole.

Despite a rigorous randomisation process, the study arms differed in education levels. Compared with the control group, more women in the intervention arm had completed high school, which could have related to their greater knowledge about LARC availability. However, the groups had equal rates of college attendance. Furthermore, a regression analysis showed that the difference in knowledge remained significant after controlling for education level.

For the purposes of research, this contraception notification was administered as a stand-alone telephone call. In considering whether to integrate this intervention into clinical care, we should note that while the intervention did significantly improve knowledge of LARC availability, $29 \%$ of those who were told LARC would be available at the time of abortion did not retain this information by the day of their visit. Clinic staff must weigh the potential benefits of advance notice of contraception against the additional telephone call time and imperfect increase in knowledge.

Our site facilitates easy access to all available forms of post-abortion contraception, which enabled us to study this intervention in the absence of competing barriers. However, post-abortion contraception is not universally accessible, and at a site with other obstacles to immediate initiation of LARC at the time of abortion, advance notice about LARC might not be as meaningful. Conversely, because our clinic has a high baseline rate of immediate post-abortion LARC, a site with lower baseline LARC uptake might be more sensitive to an intervention. Similarly, the majority of women in our study had completed some or all of college, and it is possible that our intervention might have greater impact on a less educated population.

We assessed contraceptive knowledge on arrival to the clinic appointment in order to isolate the effect of our intervention. Future studies could compare contraceptive knowledge after counselling during the visit in order to assess any benefit that advance notice confers beyond the standard of care. Grounding our study design in the transtheoretical model of health behaviour, in which change progresses through stages (including pre-contemplation, contemplation, preparation, and action), ${ }^{19}$ we hypothesised that providing patients with contraceptive options information several days prior to their abortion procedure would allow them to move from a contemplation phase to action. Studies with a larger sample size would be able to detect whether advance notice about contraception influences contraceptive choice and uptake.

Accurate contraceptive information is crucial for contraceptive decision-making. Advance notice about post-abortion contraception addresses misconceptions and allows women additional time and space to 
knowledgeably consider the most appropriate contraceptive method after abortion. In practice, advance notice of contraceptive availability and effectiveness may be integrated into routine abortion care to improve patients' contraceptive knowledge prior to the clinic visit without compromising their contraceptive autonomy.

Funding This work was supported by the Society of Family Planning Research Fund [grant number SFPRF16-8]. The trial is registered at http://clinicaltrials.gov [NCT02836561].

Competing interests None declared.

Patient consent Not required.

Ethics approval Partners Institutional Review Board.

Provenance and peer review Not commissioned; externally peer reviewed.

(c) Article author(s) (or their employer(s) unless otherwise stated in the text of the article) 2018. All rights reserved. No commercial use is permitted unless otherwise expressly granted.

\section{REFERENCES}

1 Okusanya BO, Oduwole O, Effa EE. Immediate postabortal insertion of intrauterine devices. Cochrane Database Syst Rev 2014;7:CD001777.

2 Boyd EF, Holmstrom EG. Ovulation following therapeutic abortion. Am J Obstet Gynecol 1972;113:469-73.

3 Boesen HC, Rørbye C, Nørgaard M, et al. Sexual behavior during the first eight weeks after legal termination of pregnancy. Acta Obstet Gynecol Scand 2004;83:1189-92.

4 Kavanaugh ML, Carlin EE, Jones RK. Patients' attitudes and experiences related to receiving contraception during abortion care. Contraception 2011;84:585-93.

5 Goodman S, Hendlish SK, Reeves MF, et al. Impact of immediate postabortal insertion of intrauterine contraception on repeat abortion. Contraception 2008;78:143-8.

6 Rose SB, Lawton BA. Impact of long-acting reversible contraception on return for repeat abortion. Am J Obstet Gynecol 2012;206:37.e1-37.e6.

7 Langston AM, Joslin-Roher SL, Westhoff CL. Immediate postabortion access to IUDs, implants and DMPA reduces repeat pregnancy within 1 year in a New York City practice. Contraception 2014;89:103-8.
8 Stanek AM, Bednarek PH, Nichols MD, et al. Barriers associated with the failure to return for intrauterine device insertion following first-trimester abortion. Contraception 2009;79:216-20.

9 Flamant A, Ouldamer L, Body G, et al. Rates of continuation and satisfaction of immediate intrauterine device insertion following first- or second-trimester surgical abortion: a French prospective cohort study. Eur J Obstet Gynecol Reprod Biol 2013;169:268-74.

10 Madden T, Eisenberg DL, Zhao Q, et al. Continuation of the etonogestrel implant in women undergoing immediate postabortion placement. Obstet Gynecol 2012;120:295-9.

11 Mark A, Sonalkar S, Borgatta L. One-year continuation of the etonogestrel contraceptive implant in women with postabortion or interval placement. Contraception 2013;88:619-23.

12 Thompson KM, Speidel JJ, Saporta V, et al. Contraceptive policies affect post-abortion provision of long-acting reversible contraception. Contraception 2011;83:41-7.

13 Harper CC, Rocca CH, Thompson KM, et al. Reductions in pregnancy rates in the USA with long-acting reversible contraception: a cluster randomised trial. Lancet 2015;386:562-8.

14 Rose SB, Cooper AJ, Baker NK, et al. Attitudes toward longacting reversible contraception among young women seeking abortion. J Womens Health 2011;20:1729-35.

15 Stanwood NL, Bradley KA. Young pregnant women's knowledge of modern intrauterine devices. Obstet Gynecol 2006;108:1417-22.

16 Ferreira AL, Lemos A, Figueiroa JN, et al. Effectiveness of contraceptive counselling of women following an abortion: a systematic review and meta-analysis. Eur J Contracept Reprod Health Care 2009;14:1-9.

17 Matulich M, Cansino C, Culwell KR, et al. Understanding women's desires for contraceptive counseling at the time of first-trimester surgical abortion. Contraception 2014;89:3641.

18 Eisenberg DL, Secura GM, Madden TE, et al. Knowledge of contraceptive effectiveness. Am J Obstet Gynecol 2012;206:479.e1-479.e9.

19 Prochaska JO, Velicer WF. The transtheoretical model of health behavior change. Am J Health Promot 1997;12:38-48. 